\title{
Serie $\rightarrow$ Stichwort des Monats Tracheotomierte betreuen
}

$\nabla$

Das Tracheostoma ist eine künstlich angelegte Öffnung im vorderen Bereich der Luftröhre. Es wird mit Hilfe einer Trachealkanüle offen gehalten. Indikationen für die Anlage eines Tracheostomas sind beispielsweise Tumoren im Rachenraum, Kehlkopfkarzinome, Langzeitbeatmung, Stenosen, massive Verletzungen im Gesichts- und Schädelbereich.

Nach einer Tracheotomie ist der Patient in seiner Kommunikationsfähigkeit stark eingeschränkt, da er nicht mehr sprechen kann. Um dennoch eine Kommunikation zwischen Patient und Pflegenden zu ermöglichen, ist unter anderem die Verwendung von Kommunikationstafeln hilfreich.

Unmittelbar nach einer Tracheotomie kommt eine Kunststoffkanüle mit aufblasbarer Manschette (Cuff) zur Anwendung. Die Manschette dichtet den Raum zwischen Tubus und Trachealwand ab, so dass eine Beatmung erfolgen kann und der Patient vor einer Aspiration geschützt wird. Um Druckschäden in der Trachea zu verhindern, darf die Manschette nur mit so viel Luft aufgeblasen werden, wie nötig ist, um den Raum zwischen Trachea und Tubus abzudichten. Die Überprüfung der Undurchlässigkeit erfolgt durch Auskultation der Trachea mit einem Stethoskop. Sind pfeifende oder blubbernde Nebengeräusche beim Atmen zu hören, ist dies ein sicherer Hinweis dafür, dass der Raum zwischen Tubus und Trachealwand nicht ausreichend abgedichtet ist. Damit es zu keiner Schädigung der Trachealschleimhaut kommt, kann ein spezieller Manschettendruckmesser verwendet werden.

Komplikationen nach einer Tracheotomie können sein:

- Kanülenfehllage

- Massive Blutungen und damit erhöhte Aspirationsgefahr,

- Verstopfungen der Kanüle durch Sekret

Nach einer Tracheotomie wird die Einatemluft nicht mehr ausreichend erwärmt, angefeuchtet und gefiltert, da der Nasenund Rachenraum ausgeschaltet ist. Das

\section{QUIZ \\ Menschen pflegen 3 Fragen - 3 Monate - 3 Bände}

Wir verlosen das komplette Werk „Menschen pflegen" - und so gehts: In dieser und den nächsten zwei Ausgaben von Heilberufe stellen wir Ihnen jeweils eine Frage zum Stichwort des Monats. Wenn Sie den Text sorgfältig gelesen haben, können Sie die Fragen sicherlich richtig beantworten. Diesmal lautet sie:

„Was gehört bei Tracheotomierten nicht in ein Notfallset?" a) Ambubeutel

b) $10 \mathrm{ml}$ Blockerspritze

c) Trachealspreizer

Haben Sie alle drei Antworten zusammen,

senden Sie die Lösungsbuchstaben bitte bis zum 15.12.2008 an:

Redaktion Heilberufe

Ehrenbergstr. 11 - 14, 10245 Berlin

Fax: 03020456012

Email: heilberufe@urban-vogel.de

\section{Aus: Menschen pflegen}

Band 2, Kap. A6; von Nina Kela und Norbert Matscheko.

Die drei Bände im Set: 69,95€, 2660 S., ISBN 978-3-540-69922-4

Band 1: 29,95€, $690 \mathrm{~S}$., ISBN 3-540-23507-8

Band 2: $34,95 €, 1.200 \mathrm{~S}$., ISBN 3-540-29433-3

Band 3: $29,95 €, 1.000 \mathrm{~S}$., ISBN 3-540-29435-X

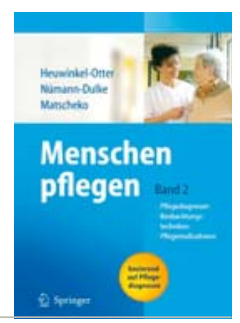

Sekret kann nicht heruntergeschluckt oder abgehustet werden. Die Folge ist eine schnelle Verlegung des Lumens der Kanüle. Deshalb erfolgt eine Anfeuchtung der Atemluft und das Sekret wird abgesaugt.

An den Platz eines tracheotomierten Patienten gehört für den Notfall (versehentliche Dekanülierung oder Verstopfung der Kanüle) ein Nothilfeset: 10-mlBlockerspritze, drei Trachealkanülen (eine größere, eine kleinere und die passende Größe) und ein Trachealspreizer.

Je nach Erkrankung werden unterschiedliche Trachealkanülen angewendet. Langzeitkanülenträger erhalten zum Beispiel Kunststoffkanülen oder Silberkanülen mit Sprechventil. Diese bestehen aus einer Außen- und einer Innenkanüle („Seele"). Bei Verstopfung durch Sekret kann die Innenkanüle leicht herausgenommen werden, ohne dass dabei die eigentliche Kanüle aus dem Tracheostoma entfernt werden muss. Der Einsatz einer Sprechkanüle sollte so bald wie möglich erfolgen, damit der Patient wieder sprechen kann. 